\title{
Contribución de la política alimentaria al descenso de la desnutrición en el Ecuador
}

The Contribution of Food Policy to the Descent of Malnutrition in Ecuador

Susana Olarte

Universidad de las Américas,

Quito, Ecuador

olartesusana@hotmail.com

Fecha de recepción:

5.3.15

Fecha de aceptación:

30.6.15

\begin{abstract}
Resumen
La desnutrición en el Ecuador ha descendido sustancialmente desde 2005. El grupo más afectado por la desnutrición es el de la población rural más pobre, que compra la mayor parte de los alimentos que consume. Para facilitar el acceso económico a los alimentos se ha tratado de aumentar los ingresos de los grupos más vulnerables y estabilizar los precios. Se han amentado los recursos destinados a la compra de alimentos por las políticas de transferencias directas a los grupos más vulnerables, pero los precios no han dejado de aumentar. Resulta adecuado entonces considerar la diversificación de las políticas aplicadas y potenciar las ya existentes para lograr impactos más intensos que sean además sostenibles en el tiempo. El abordaje practicado en el artículo sigue en líneas generales el enfoque del Amartya Sen.

Palabras clave: Política alimentaria, Desnutrición, Seguridad alimentaria, Transferencias directas, Ecuador.
\end{abstract}

\footnotetext{
Abstract

Malnutrition in Ecuador has declined substantially since 2005. The group most affected by malnutrition is the poorest rural population, which buys most of its foodstuffs. To facilitate economic access to food the government
} 
has resorted to upgrade the incomes of the most vulnerable groups together with prices stabilization. However policies of income transfers for food purchases have not prevented a steady increase in prices. It would then be appropriate to consider the diversification of policies and strengthen existing ones to achieve greater impacts that are also sustainable over time. The approach conducted in this article follows in its broad guidelines that of Amartya Sen.

Keywords: Food policy, Malnutrition, Food Security, Direct cash transfers, Ecuador.

\section{Introducción}

Unacomparacióndelainformaciónsobreprevalenciadesubalimentaciónydeladesnutrición en Ecuador muestra cómo desde 1992 y hasta 2012, el promedio es siempre mayor en este país que la media mundial y la latinoamericana (Gráfico 1) aunque la tendencia es decreciente en ambos casos, sobre todo a partir de 2005. Desde 1992 y hasta 1997 se registra una reducción progresiva de la subalimentación, pero a partir de 1998 se advierte un repunte interpretado como efecto de la profunda crisis económica y financiera nacional que estalló en ese año (Espinoza 2005) llevó en 1999 al desempleo y subempleo al $16 \%$ y $57 \%$ de la población, respectivamente, y sumió a las dos terceras partes de los ecuatorianos en la pobreza (Cerdas et al. 2006). Desde que en 2004 comenzaran a registrarse los primeros repuntes sostenidos de los principales indicadores macroeconómicos, se aprecia una evolución decreciente de la subalimentación en el Ecuador. Al analizar la desnutrición se observa la misma tendencia: una reducción progresiva de los niveles de la prevalencia de la desnutrición, con un repunte sustancial desde 1998 y hasta 2003 vinculado a la crisis de finales de la década de 1990 (De la Torre y Mascaró, 2011). La tendencia revierte a partir de 2004, generándose una reducción de la subalimentación hasta 2012 del 7,6\% (1.213.505 personas) y de la desnutrición del 5,9\% (1.024.030 personas).

La población rural es el grupo más afectado por la subalimentación y la desnutrición, con los peores índices de consumo de proteínas, hierro, zinc, vitamina A, y carbohidratos (Gráfico 2). En consecuencia se registran en el ámbito rural los mayores porcentajes de retardo en la talla, que alcanza al 29\% (INEC, 2013). Al analizar la relación entre las condiciones nutricionales y la pobreza a través de una muestra sobre anemia, consumo de zinc y de vitamina A, se comprueba que los datos más insatisfactorios se registran en los grupos más pobres (INEC 2013). La deficiencia nutricional se traduce en un mayor retardo en la talla en los estratos más pobres (Gráfico 3). 
Gráfico 1. Prevalencia de la subalimentación y de la desnutrición en porcentaje (1992-2012)

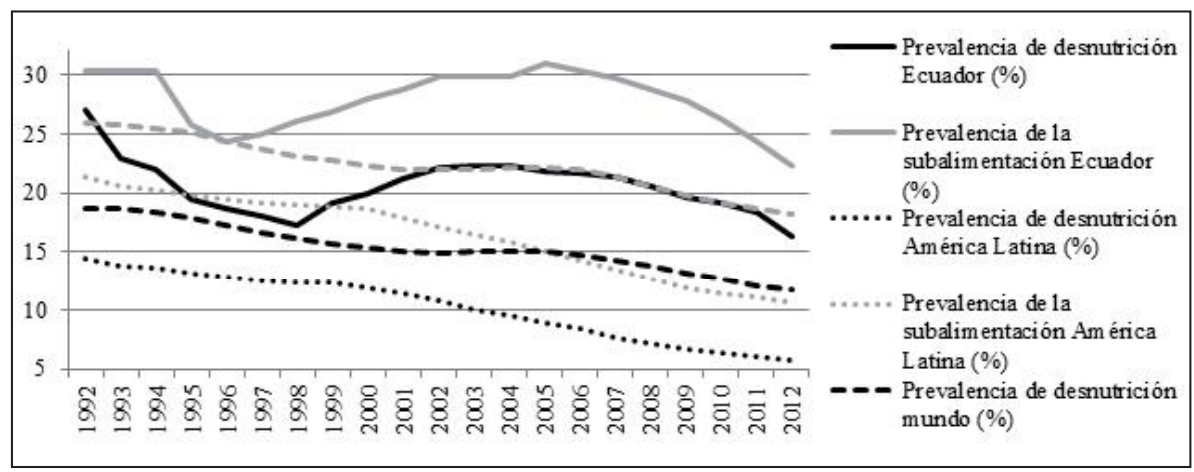

Fuente: FAOSTAT, 2014 y Banco Mundial, 2014

NOTA: El año indicado se refiere al año central de un promedio de 3 años

Gráfico 2. Prevalencia del consumo inadecuado de nutrientes $\left({ }^{\star}\right)$ por subregión, 2013

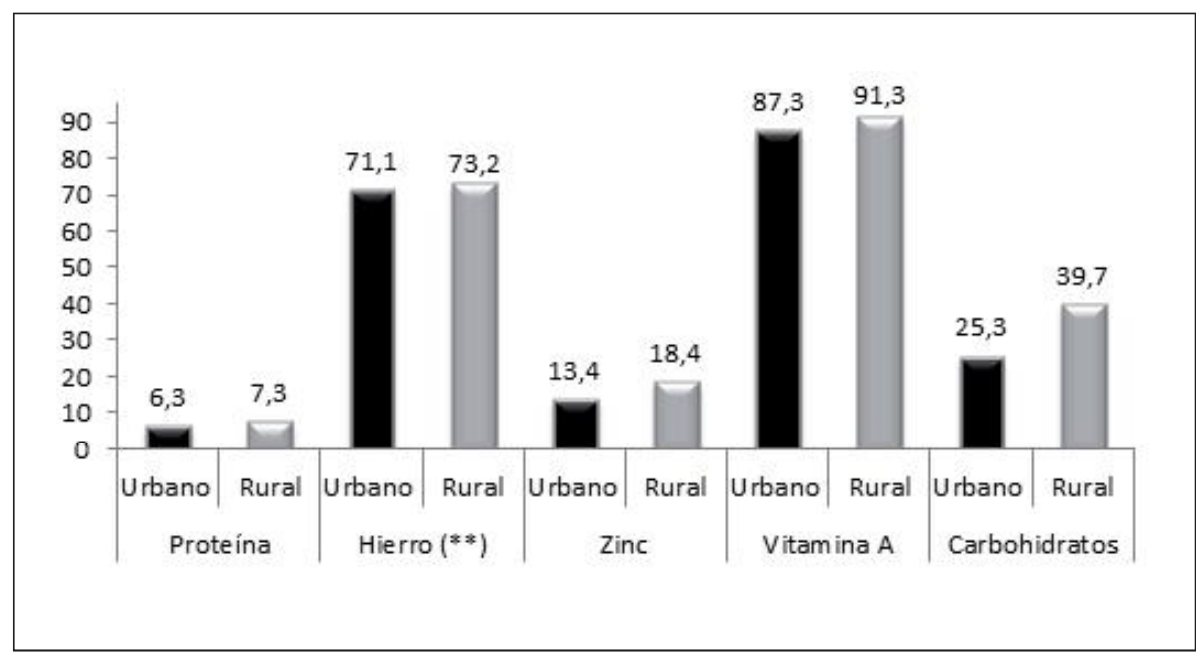

( ( Con base en el Requerimiento Promedio Estimado de las Referencias de Ingesta Dietética del Instituto de Medicina de los EEUU.

$(\star \star)$ Asumiendo una biodisponibilidad del 8\%.

Fuente: ENSANUT 2011-2013, 2013 
Gráfico 3. Ecuador: retardo en la talla, por quintil ( $\left.{ }^{\star}\right), 2013$

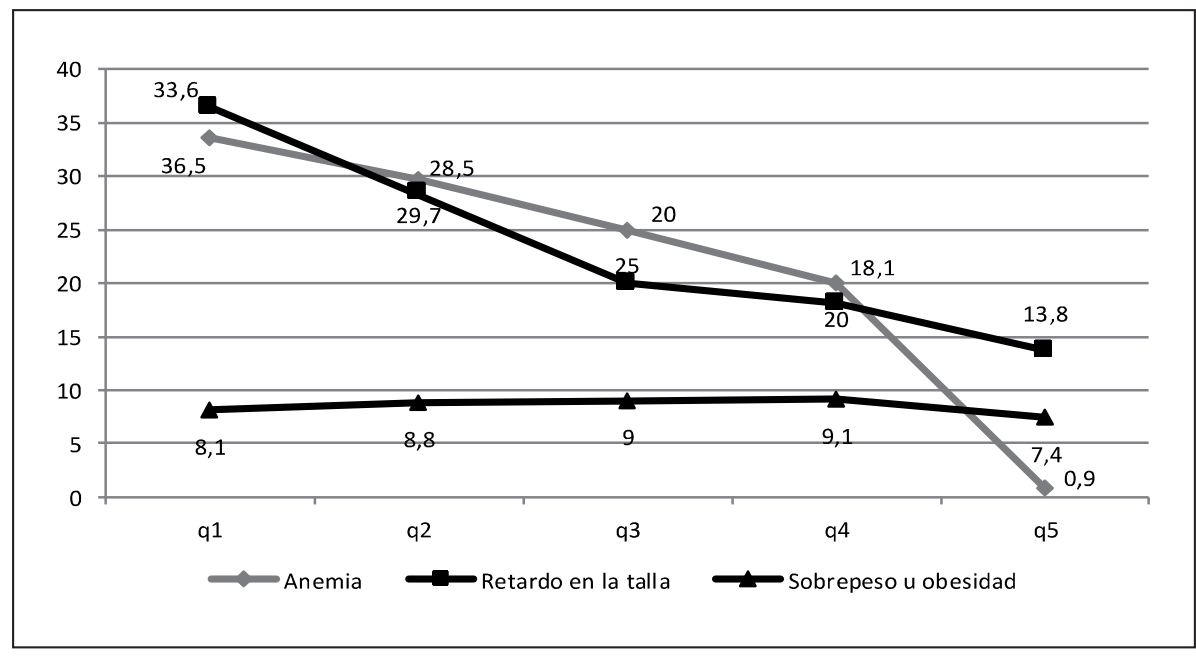

(*) Los quintiles dividen la población en cinco grupos en función de su nivel de ingresos. Fuente: ENSANUT 2011-2013, 2013

La mayoría de las personas incluidas en el grupo de mayor vulnerabilidad no produce los alimentos que consume, sino que los adquiere en relaciones mercantiles. Es necesario indagar, en consecuencia, el papel desempeñado por las políticas públicas en materias de seguridad alimentaria en la reducción de esos indicadores que se advierte en los años recientes. Con tal fin se analiza en las secciones siguientes las políticas de reducción de precios y las estrategias de aumento de ingresos, y el impacto de en la capacidad de compra de alimentos de los grupos rurales más pobres.

\section{Las causas de la desnutrición de la población rural más pobre en el Ecuador}

Para realizar un análisis de las causas específicas de la desnutrición de los más pobres en el ámbito rural en Ecuador, se atiende al trabajo del Premio Nobel de Economía Amartya Sen. Sen considera que la desnutrición se debe a una falta de acceso a los alimentos, que a su vez viene dado por la titularidad sobre los alimentos, que puede tenerse o no. La titularidad sobre el alimento se refiere a la manera en que puede accederse a él: comprándolo, recibiéndolo a modo de donación, o produciéndolo. Definió Sen tres tipos de titularidades: las titularidades de intercambio de alimentos por dinero, las titularidades transferidas, como las obtenidas por herencias o por percepciones dadas por el Estado o la comunidad, y las basadas en la producción propia (Sen, 1981). 
Los grupos más vulnerables en Ecuador, es decir, aquellos que viven en el ámbito rural y están en el decil 1 de pobreza, compran sus alimentos. El porcentaje de la producción agropecuaria que se compra oscila en torno al $75 \%$ en promedio, como puede verse en el Gráfico 4, elaborado con base a la información obtenida en las entrevistas en las comunidades ruralesa ubicadas en el decil 1 y a las líneas de base de proyectos tomados como fuente de datos, información detallada en el Anexo. Precisamente porque la mayor parte de los alimentos para el consumo se compra, se concentra esta investigación en el análisis de las dificultades experimentadas para comprarlos.

Gráfico 4. Porcentaje de los alimentos que se consumen fuera de las comunidades

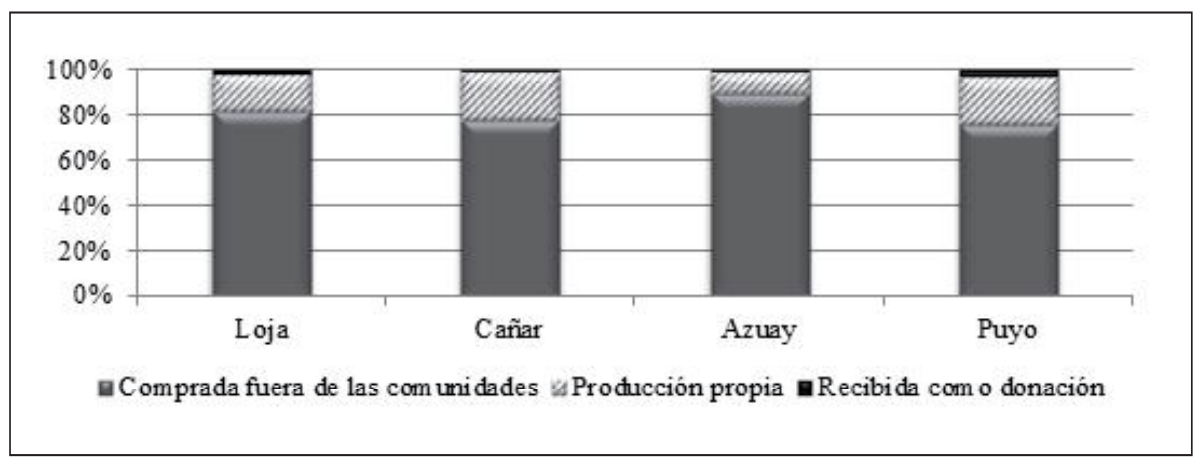

Fuente: Elaboración propia con datos procedentes de las entrevistas a las organizaciones y miembros de las comunidades en las que se ejecutaron los proyectos, y de las memorias de los proyectos (ver detalle en Anexo).

El análisis de las dificultades de los grupos más vulnerables para alcanzar la titularidad económica sobre los alimentos implica indagar sobre las dificultades que se enfrentan para comprarlos. Para ello es preciso valorar si existe una brecha entre el precio de los alimentos y los ingresos del potencial comprador, sea porque el precio se incrementa de manera sustancial año a año, o porque los ingresos del demandante no aumentan lo suficiente. De acuerdo a lo que se desprende del Gráfico 5, en el decil 1 el índice de precios de los alimentos creció más que el índice de ingresos, generando una caída en el acceso económico a los alimentos.

Corresponde en consecuencia enfocar las políticas aplicadas al respecto, tanto las dirigidas a la reducción y el control de los precios, como al aumento de los ingresos. En el primer caso es preciso analizar si el aumento y variabilidad de los precios tiene lugar en los alimentos de producción nacional o en los importados.

En el Gráfico 6 se aprecia que tanto la cantidad como los precios de la producción interna presentan índices de variabilidad muy superiores a los promedios anuales mun- 
diales. En tanto al efecto de los precios internacionales, como se observa en el Gráfico 7 , es muy elevado el nivel de dependencia de las importaciones, tomando como ejemplo el dato disponible de los cereales del Ecuador respecto al promedio mundial. Esto hace que el país sea mucho más vulnerable ante las subidas continuadas y generalizadas de los precios al consumo de los alimentos registradas desde el año 2000 (Herrero, 2014). Se aprecia asimismo una elevada volatilidad de los precios nacionales, superior a la media internacional y una gran dependencia del exterior acompañada de un aumento del precio internacional de los alimentos.

Gráfico 5. Ecuador: IPC de los alimentos e ingresos del decil 1 en el ámbito rural $(2000=100)$

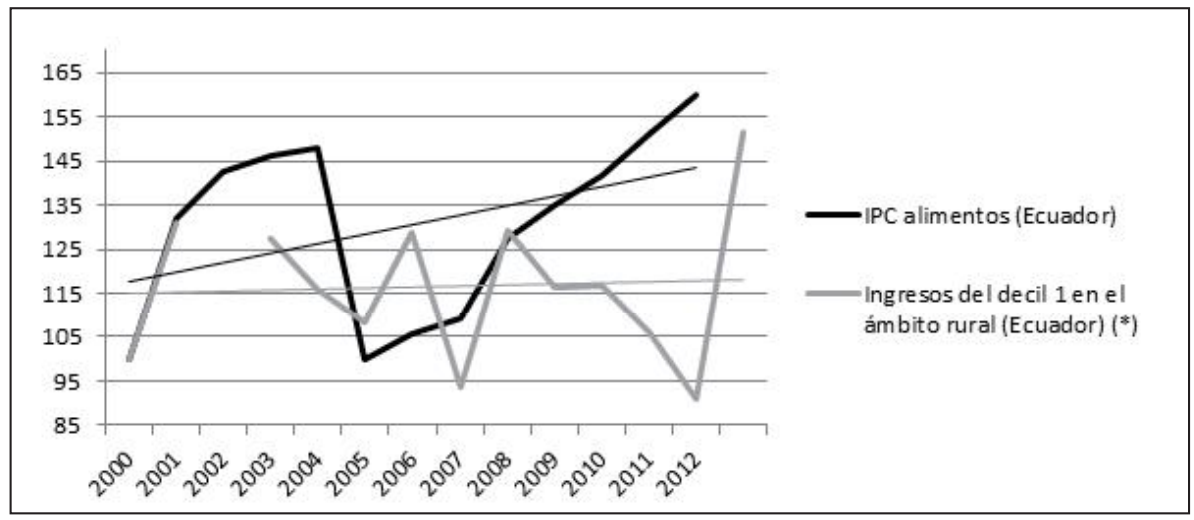

NOTA: El descenso en 2004 responde a un recálculo de la base para el cálculo del IPC (INEC, 2014). Fuente: IPC de los alimentos en Ecuador en ENSANUT 2011-2013, 2014. Ingresos en Encuesta Urbana de Empleo y Desempleo - INEC 2000-2013 en SIISE, 2014

Gráfico 6. Variabilidad de la producción de los alimentos y de los precios internos del Ecuador y el Mundo (1991-2013)

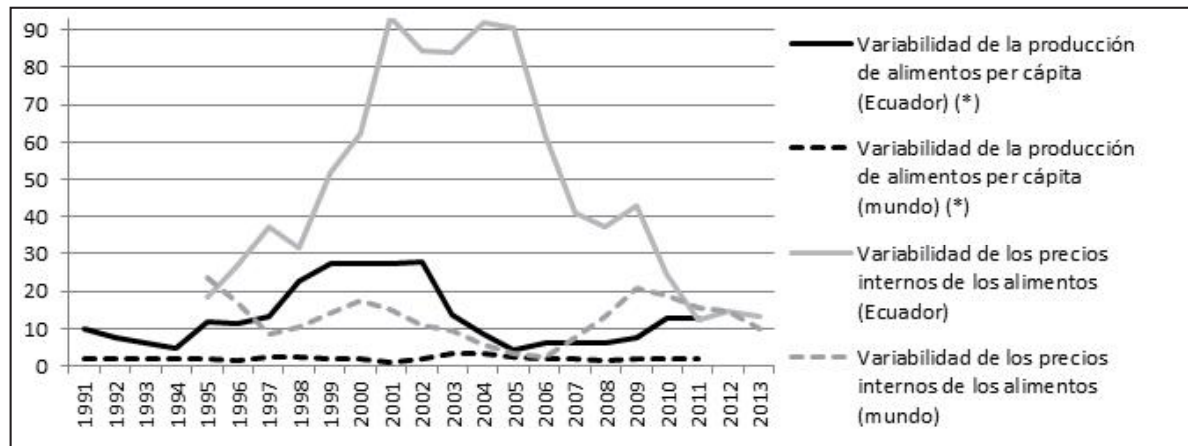

( ${ }^{\star}$ I\$ por persona constante 2004-2006

Fuente: FAOSTAT, 2014 
Gráfico 7. Dependencia de las importaciones de los alimentos del Ecuador e índice del precio internacional de los alimentos (1991-2009)

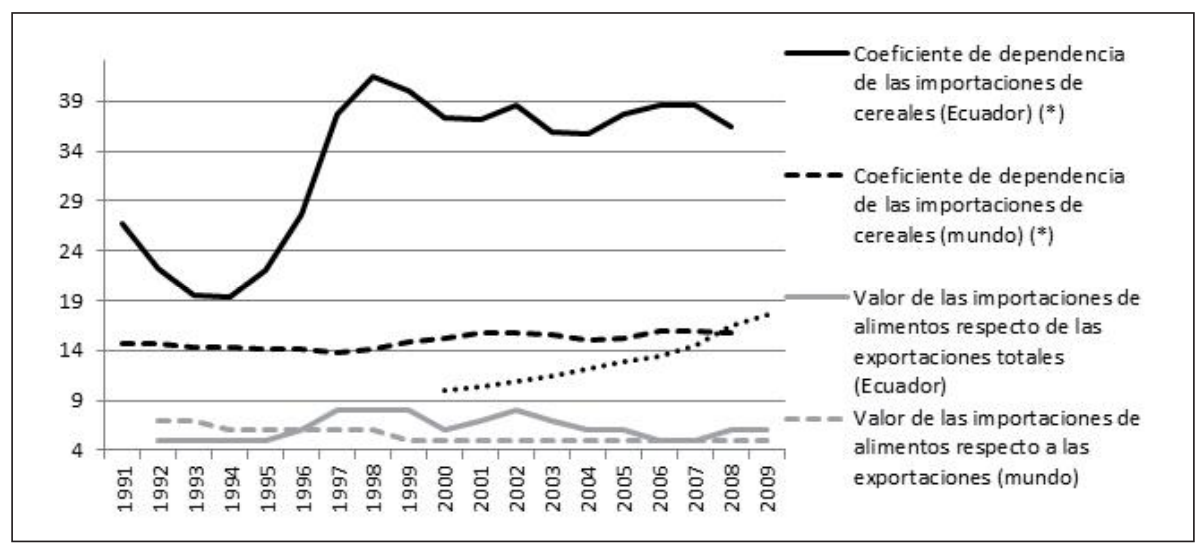

(*) En porcentaje. El año indicado se refiere al año central de un promedio de 3 años Fuente: FAOSTAT, 2014

\section{Estrategias aplicadas para controlar los precios de los alimentos}

La estabilización de los precios y la fijación de límites pueden alcanzarse a través de la aplicación de varias políticas. Ecuador optó por el control de precios de manera directa y el aumento de la oferta de alimentos. En ambos casos, y al no generarse escasez por la fijación de los precios, el resultado esperado sería un aumento de la cantidad de alimentos producida, con un efecto como el que se indica en el Gráfico 8. El aumento de la cantidad de alimentos producida puede sustituir importaciones, estabilizar la producción y los precios e incluso aumentar su aporte a la exportación de alimentos.

Gráfico 8. Movimiento de la curva de la oferta para aumentar la producción y reducir los precios.

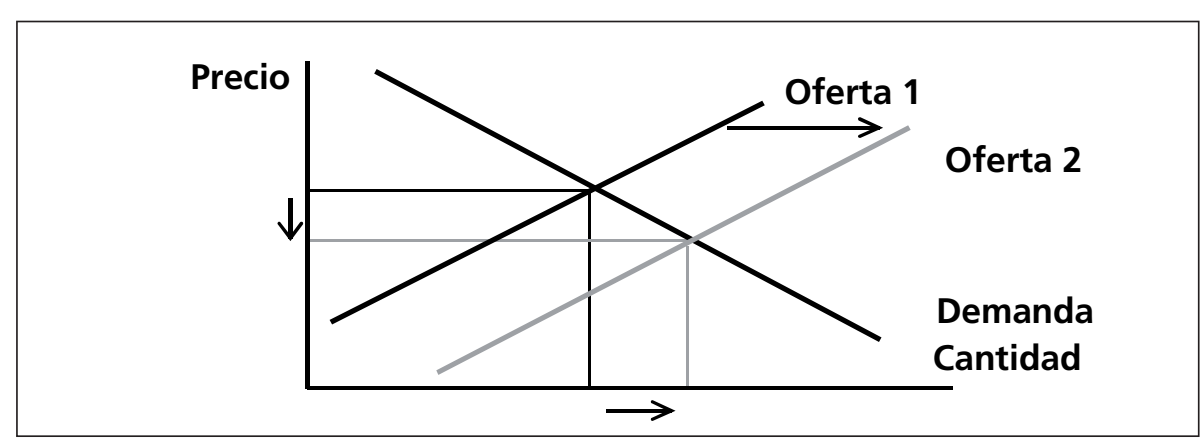

Fuente: Elaboración propia 
En cuanto al control de los precios, en diciembre de 2012, la Ley Orgánica de Comercialización y Abastecimiento Alimentario, incluye en el Artículo 61, 62, 63 y 64 la posibilidad de crear precios oficiales, de sustentación, referenciales y bandas de precios (República del Ecuador, 2013). En febrero de 2013 se firmó el Decreto Ejecutivo 1438 que estipuló la creación de precios referenciales de los principales alimentos, que los mercados mayoristas debían observar (MIPRO, 2013).

En cuanto al aumento de la oferta, se optó por el desarrollo de varios programas de mejora de los insumos agropecuarios en el marco de un incremento de la inversión pública (Gráfico 9). El aumento responde a un presupuesto nacional cada vez mayor apuntalado por la recuperación del control nacional de la mayor exportación del Ecuador -el petróleo-, y sus altos precios registrados durante el periodo (BCE, 2014).

El aumento de la inversión privada se acompañó de un aumento de los principales indicadores macro económicos en un clima de sostenibilidad política y social (BCE, 2014). El resultado del aumento de la inversión pública y de la privada fue un tímido aumento de la cantidad de alimentos producida y un mayor rendimiento de la producción de alimentos en el Ecuador, superior a la media mundial, como se indica en los gráficos 10 y Gráfico 11.

Sin embargo los resultados de las políticas de control de precios y de aumento de la oferta de alimentos no han sido los deseados. El tímido repunte de la producción y el incremento de la rentabilidad no han podido generar un descenso real de los precios, estabilidad duradera, sustituir las importaciones o aumentar las exportaciones de los alimentos de manera sostenible. Ha sido mayor el peso del aumento de la demanda de alimentos por el incremento de la población, el aumento de los salarios, el descenso del desempleo y el efecto de la inflación generalizada (BCE, 2014).

\section{Gráfico 9. Crecimiento anual del Presupuesto General del Estado sector agropecuario.}

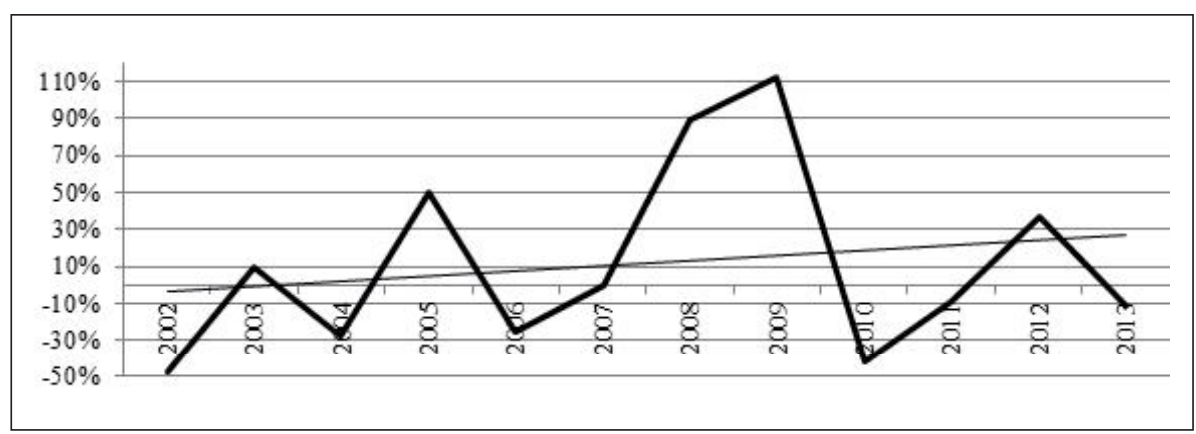

Fuente: Sistema Integrado de Gestión Financiera - Ministerio de Finanzas en SIISE, 2014 
Gráfico 10. Índice de producción bruto per cápita de alimentos en el Ecuador (base 2004-2006 $=100$ )

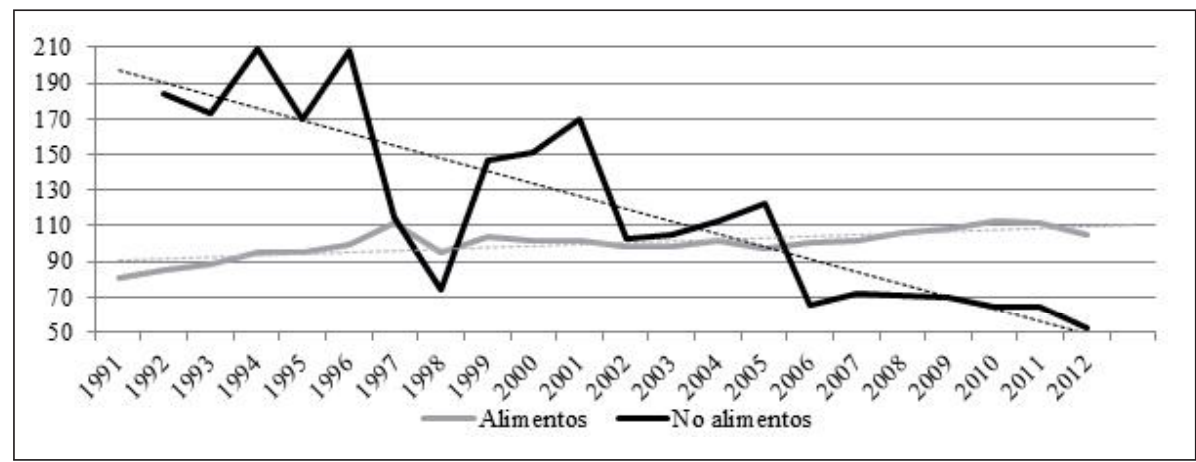

Fuente: FAOSTAT, 2014

Gráfico 11. Rendimiento de la producción de cereales en Ecuador y el mundo (1990-2012)

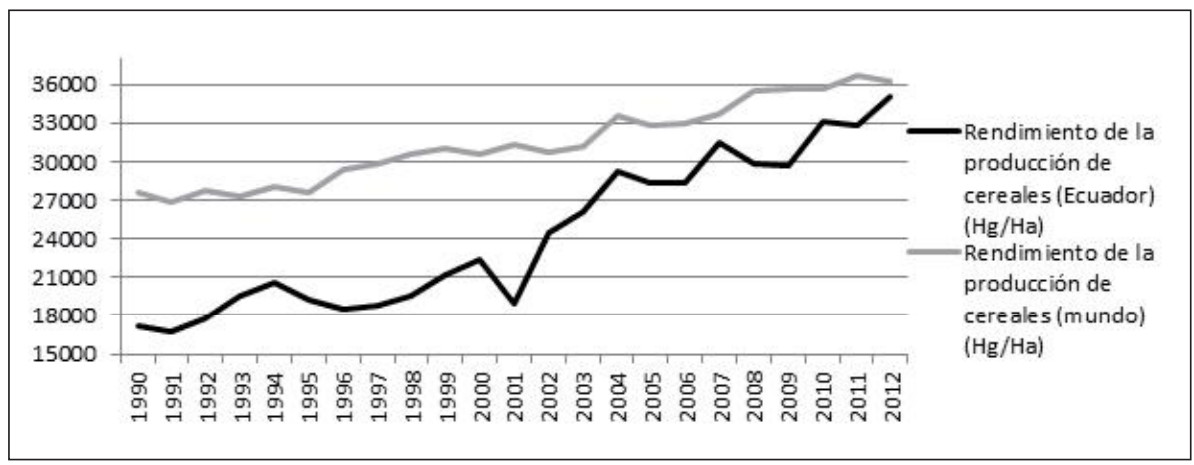

Fuente: FAOSTAT, 2014

\section{Políticas para aumentar los ingresos}

Schady y Rosero (2008) confirman que el aumento de los ingresos tiene una relación directa con el incremento de los recursos destinados a los alimentos, lo que incide positivamente en las condiciones nutricionales. Según la ECV 2005-2006, el $90 \%$ de las remesas de dinero provenientes de residentes en el exterior se destinaron a educación, salud, alimentos y arriendo. Tal y como se aprecia en el Gráfico 12, el incremento de los ingresos de los proyectos desarrollados en el Ecuador en el ámbito rural tomados como referencia fue en promedio del $25 \%$. De ese $25 \%$, un tercio se destinó a comprar más alimentos y de mejor calidad. 
Desde el Estado ecuatoriano se han aplicado programas para el aumento de los ingresos de los estratos más bajos en el ámbito rural que se dividen entre los que buscan un aumento directo de los ingresos o de la reducción del gasto en alimentos, y los que buscan un incremento sostenible de los ingresos.

Gráfico 12. Destino del aumento de los ingresos en el decil 1 en el ámbito rural

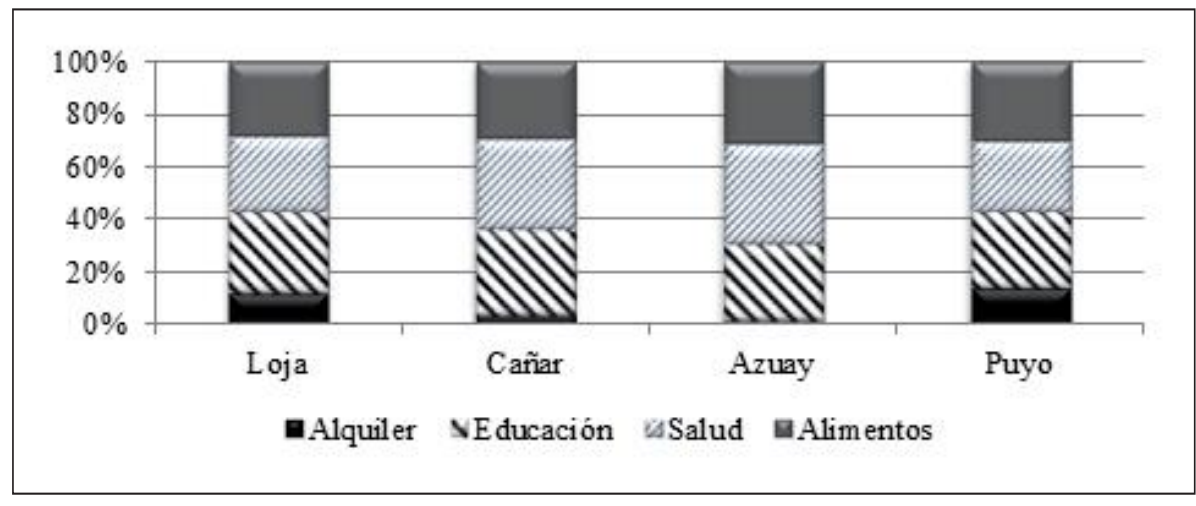

Fuente: Elaboración propia con datos procedentes de las entrevistas a las organizaciones y miembros de las comunidades en las que se ejecutaron los proyectos, y de las memorias de los proyectos.

En materia de políticas de incremento de los ingresos de la población afectada, destaca el Bono de Desarrollo Humano (BDH). Consiste de una transferencia monetaria mensual de 50 dólares a las madres de familia, a los mayores de 65 años, y a las personas con más de un $40 \%$ de discapacidad que están en los dos grupos más pobres. La transferencia a la familia está condicionada: si el hogar tiene niños menores de 6 años, deben participar de controles de salud al menos dos veces al año; si el hogar tenía un niño entre 6 a 15 años, se condicionaba la transferencia a que estuvieran matriculados en la escuela y que asistieran a las clases por lo menos el 90\% de los días (MIES, 2014). El programa fue creado en 1998 con el nombre de Bono Solidario, como un subsidio directo que tenía el objeto de proteger el consumo mínimo de las personas pobres del país que se verían afectadas por una eliminación de otros subsidios. Posteriormente, y dada la crisis económica política y económica que afectó al país al final de la década de 1990 (Espinoza, 2005), pasó a ser la principal herramienta del gasto social del país para combatir los efectos de la crisis y proteger a la población más vulnerable (MIES, 2014). En lo que toca a subsidios a los precios, destaca el Programa Socio Ahorro (PSA) impulsado en 2008, que contempló un descuento del 8\% sobre 60 dólares de la cesta de la compra, del que se beneficiaban el mismo grupo de personas que del $\mathrm{BDH}$. 
Atendiendo a los datos registrados en el Gráfico 13, desde 2004 el número de personas con $\mathrm{BDH}$ no ha dejado de crecer. Casi la tercera parte de los nuevos ingresos procedentes del Bono se destinaba a alimentos, es decir, de los 50 dólares recibidos, 15 se destinaban a comida. Esta cantidad estimada ha ido aumentando conforme aumentaba el BDH. A este dato se le ha de añadir, desde 2008, el 8\% de descuento sobre los 60 dólares gastados en la cesta de la compra, que suponen 4,8 dólares. El total es de 19,8 dólares, del que disponían en 2013 1.719.504, personas. Suponen 651.032 personas más que en 2004, cuando comenzó el descenso de la desnutrición y de la subalimentación. Esta cifra representa la mitad de las personas que han dejado de estar en condición de subalimentación y desnutrición desde 2004. La información disponible, sin embargo, no permite discernir entre áreas rurales y urbanas.

Gráfico 13. Personas con recursos del Estado que destinaban a alimentos (subsidio directo e indirecto)

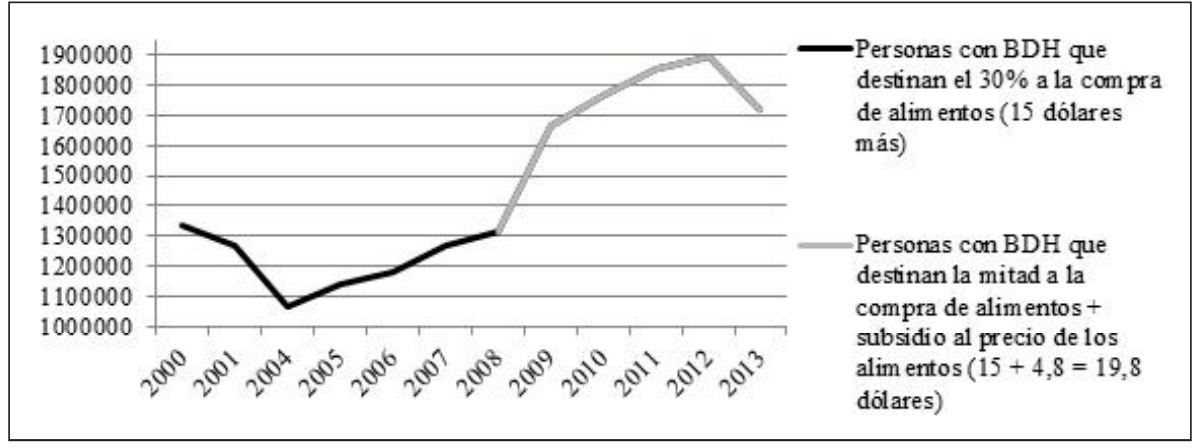

Fuente: MIES, 2014

En cuanto a las políticas generadoras de ingresos sostenibles de las que se pudieron beneficiar en el sector rural más pobre destacan el Crédito de Desarrollo Humano $(\mathrm{CDH})$, los Programas del Servicio Ecuatoriano de Capacitación Empresarial (SECAP) y los programas "Nutriendo el Desarrollo" e "Hilando el Desarrollo". El Crédito de Desarrollo Humano busca mejorar los niveles de vida de los beneficiarios del BDH para incorporarlos al tejido socio productivo nacional. Son créditos ligados al Programa Productivo Solidario de hasta 840 dólares a dos años, con una tasa de interés de 5\%, sin garantías. En 2011, el 7,7\% de los hogares que había recibido el BDH recibió el $\mathrm{CDH}$, con una cobertura de 142.917 personas. El Programa del Servicio Ecuatoriano de Capacitación Empresarial (SECAP), desde 2000 y hasta 2010, había capacitado a 885 mil personas (SIISE, 2014a). El programa "Nutriendo el Desarrollo" a cargo del Ministerio de Agricultura, Ganadería, Acuacultura y Pesca (MAGAP), busca fomentar la producción local de leche para abastecer a los programas de alimentación del Estado. El 
objetivo principal es fomentar la inclusión económica de los pequeños productores de leche, así como la coparticipación en forma asociada en la industrialización de este producto. El programa capacita a los productores y garantiza la compra de leche a centros de acopio que fomenta la Asociación de Ganaderos de la Sierra y el Oriente (AGSO). En 2007 participaban 1.420 pequeños productores, aumentándose la cobertura hasta alcanzarse los 3.190 en 2010 (SIISE, 2014).

El Programa “Hilando el Desarrollo”, del Ministerio de Inclusión Económica y Social (MIES), se inició en 2007 para vincular a los pequeños tejedores a espacios mayores de confección que puedan ofrecerles mejores oportunidades económicas a medio y largo plazo. En 2007 participaban 220 pequeños productores, aumentándose la cobertura hasta alcanzarse 1.881 personas en 2010 (SIISE, 2014).

Se carece de información acerca de la evolución de los ingresos de las familias participantes en estos programas, lo que no permite calcular el aumento de los recursos destinados a la compra de alimentos, aunque pudiera considerarse que efectivamente tuvo lugar.

\section{Conclusiones}

El Ecuador ha experimentado un descenso de la desnutrición y de la subalimentación de manera sostenible desde 2004 y hasta 2012. El colectivo más afectado por la desnutrición y la subalimentación son las personas en el campo que pertenecen al estrato más pobre. Estas familias compran la mayor parte de los alimentos, es decir, tienen alimentos porque acceden económicamente a ellos. Durante el periodo analizado la capacidad de las familias más pobres en el ámbito rural para comprar alimentos ha descendido en la medida en que los precios de los alimentos han aumentado más que los ingresos.

En general las políticas aplicadas para reducir y estabilizar los precios y aumentar los ingresos se consideran adecuadas en tiempo y forma, máxime teniendo en cuenta el poco tiempo que llevan ejecutándose. Se consideraría adecuado en adelante que fueran más agresivas, diversas y duraderas, de cara a lograr resultados más intensos y duraderos.

Las políticas destinadas a reducir y estabilizar el precio de los alimentos se concretaron en el aumento de la oferta de alimentos y en el control mediante precios de referencia. El aumento de la producción fue insuficiente para cubrir la nueva demanda generada por el aumento de la población y el incremento de la renta media.Valdría la pena considerar la apuesta por un incremento de la producción de alimentos mucho más agresivo que permitiera incluso sustituir importaciones y reducir la vulnerabilidad frente a las oscilaciones de los precios y los volúmenes de producción externos. De igual manera el aumento de la producción de alimentos podría haber contribuido a la balanza comer- 
cial a través del aumento de las exportaciones y potenciar la estructura productiva del Ecuador. Debía tratarse de un incremento de la producción final calculado que tuviera en cuenta la evolución de la demanda interna y externa. Con ese objetivo, se tendría que haber diseñado una estrategia de inversión, producción y comercialización nacional e internacional previo con metas cuantificables, indicadores cuantitativos y cualitativos y fuentes de verificación.

En tanto a la política de control de los precios se aprecia una mayor necesidad de contar con una difusión entre los consumidores y de una atención por parte de las autoridades más intensa del cumplimiento de la toma como referencia de los precios indicados por el gobierno por parte de los mayoristas. Además se requeriría de un tratamiento integral del control de precios que trate también los insumos y las materias primas necesarias para la producción, pudiendo valorar políticas de subvención o recompra por parte del Estado. De igual manera, habría sido necesario considerar medidas destinadas al control de los precios de las importaciones a través de la imposición de aranceles.

Para incrementar los ingresos de las familias más pobres se optó por políticas de aumento directo de los ingresos y subvenciones a la canasta de alimentos, y políticas indirectas de incremento de los ingresos, a través de la mejora del acceso al crédito y del conocimiento para la generación de recursos.

La política de ingresos directos y de subvenciones a la canasta de alimentos dirigidas a los colectivos más pobres generó un incremento sustancial de los recursos destinados a los alimentos en términos relativos, e insuficiente en términos absolutos. Si bien no se puede distinguir entre los que recibieron el dinero y la subvención en el campo o en la ciudad, todos estaban en los estratos más pobres, con lo que se estima un impacto directo y positivo sobre el grupo que reside en el ámbito rural y está en el decil más pobre. La vulnerabilidad de los grupos a los que se dirigen los bonos y los subsidios y el adecuado control y gestión por parte del gobierno invitan a continuar con su práctica, si bien se extraña un mayor análisis no sólo sobre el destino de esos recursos, como son los alimentos o las medicinas, sino del impacto que generan sobre la condición de nutrición, salud, etc. que pueden experimentar las familias beneficiarias.

En tanto a los programas que tienen como objetivo la generación de ingresos de manera sostenible, lo que podía redundar en una mejor capacidad económica para la compra de alimentos, no se cuenta con un registro del impacto real que han tenido dichos programas. En general la meta ha sido cumplir con una serie de actividades vinculadas sobre todo a la capacitación, es decir, no se ha trabajado por resultados. Se aprecia una clara necesidad de planificar los procesos para la generación de ingresos de manera sostenible por objetivos, es decir, teniendo como metas medibles y cuantificables aquéllas relacionadas con los beneficiarios de los procesos, en vez de con los actos de los ejecutores de 
los programas.Valdría la pena entonces reforzar este tipo de programas por su capacidad para generar resultados sostenibles en el tiempo, precisamente por la condición de aislamiento y marginalidad en la que se encuentran los colectivos rurales tradicionalmente afectados por la desnutrición.

\footnotetext{
${ }^{1}$ La subalimentación hace referencia a una ingesta por debajo del mínimo recomendado por la OMS (FAO, 2008).

${ }^{2}$ El nivel de desnutrición mide el "[p]ercentage of the population whose food intake is insufficient to meet dietary energy requirements continuously. Data showing as 2.5 signifies a prevalence of undernourishment below 2,5[\%]" Banco Mundial (2014).

${ }^{3}$ Los proyectos seleccionados para el análisis son "Fortalecimiento de la migración responsable entre Cañar y Madrid a través de la prestación de servicios de información, orientación y asesoramiento y el apoyo a iniciativas productivas de familias transnacionales (2010-2012)", "Fortalecimiento del tejido socio-económico en comunidades rurales en situación de extrema pobreza y focos de migración en las provincias de Loja y Azuay (2009-2011)", "Inserción socioeconómica de familias en situación de extrema pobreza en comunidades rurales en la provincia de Loja (2009-2011)" "Mejora de las condiciones socioeconómicas de las familias en situación de pobreza en la zona rural de la provincia de Loja (2009-2011)", "Fortalecimiento del tejido socio-económico en comunidades rurales en situación de extrema pobreza y focos de migración en zonas fronterizas de Ecuador y Perú (2009-2010)" y "Fortalecimiento de las capacidades de respuesta ante desastres naturales y mejora de abastecimiento de agua de cuatro comunidades indígenas Zápara en el Cantón Puyo (2008-2010)".
} 


\section{Anexo}

Los datos sobre el tipo de acceso a los alimentos de las familias que residen en el ámbito rural y están en el decil1 de pobreza se obtuvieron de las propias comunidades. Para ello se visitaron 56 comunidades de las provincias de Azuay, Cañar, Loja y Pastaza en las que se realizaron 483 encuestas. Una de las preguntas de las encuestas realizadas era la siguiente:“¿Usted compra sus alimentos, los produce, o se los entregan?”. Las entrevistas se realizaron en el periodo comprendido entre febrero de 2009 y diciembre de 2011 con el apoyo de varias organizaciones de la sociedad civil. Se recoge en la siguiente tabla el detalle de las encuestas.

Tabla 1. Relación de las CMA visitadas y de las entrevistas con organizaciones que trabajan en las comunidades

\begin{tabular}{|l|c|c|l|}
\hline País & Comunidades & Encuestas & Organización \\
\hline Azuay & 4 & 26 & Fundación Paul Rivet \\
\hline Cañar & 32 & 290 & Fundación Nuevos Horizontes \\
\hline \multirow{3}{*}{ Loja } & 6 & 68 & Unión Popular de Mujeres de Loja (UPML) \\
\cline { 2 - 4 } & 5 & 52 & $\begin{array}{l}\text { Fondo Ecuatoriano Populorum Progressio } \\
\text { (FEPP) }\end{array}$ \\
\cline { 2 - 4 } & 4 & 26 & Fundación GRATOS \\
\hline Pastaza & 5 & 21 & Fundación Altrópico \\
\hline
\end{tabular}

Fuente: Elaboración propia

Además, se incluyeron los resultados de las líneas de base de dos proyectos de cooperación que recogían la misma pregunta de “¿Usted compra sus alimentos, los produce, o se los entregan?” en su diagnóstico inicial. Participaron en la encuesta 450 personas. Los proyectos eran "Fomento de la migración responsable entre Cañar y Madrid a través de la prestación de servicios de información, orientación y asesoramiento y el apoyo a iniciativas productivas de familias transnacionales", ejecutado en 2007 por la Fundación Nuevos Horizontes, y el proyecto "Fortalecimiento del tejido socio-económico en comunidades rurales en situación de extrema pobreza y focos de migración en las provincias de Loja y Azuay (2009-2011)" ejecutado por la Fundación Nuevos Horizontes, y la Fundación FEPP. 


\section{Referencias bibliográfícas}

Banco Central Ecuatoriano -BCE- (2014). Base de datos http://www.bce.fin.ec/ index.php/informacion-estadistica-1 accesado el 6 de enero de 2015.

Banco Mundial (2014) Base de datos http://datos.bancomundial.org/indicador/ NY.GNP.PCAP.CD accesado el 6 de enero de 2015.

Calero, Carla (2011). Seguridad alimentaria en Ecuador desde un enfoque de acceso a alimentos. Quito: FLACSO Ecuador.

Cerdas, Eduardo, Jiménez, Federico y Valverde, Miriam (2006) Crisis de Ecuador en los años 1999-2000, Quito UNED.

De la Torre, Augusto y Mascaró, Yira. (2011). La gran crisis ecuatoriana de finales de los noventa. Quito: CORDES.

Encuesta Nacional de Salud y Educación -ENSANUT- (2013). Base de datos. http://www.ecuadorencifras.gob.ec/salud-salud-reproductiva-y-nutricion/ accesado el 6 de enero de 2015.

Espinoza, Roque (2005). La Crisis Económica Financiera Ecuatoriana de finales de siglo y la dolarización.http://www.uasb.edu.ec/padh/ accesado el 6 de enero de 2015.

Estadísticas de la Organización de las Naciones Unidas para la Alimentación y la Agricultura -FAOSTAT- (2014). Base de datos. http://faostat.fao.org/site/339/ default.aspxaccesado el 6 de enero de 2015.

FAO (2008) El estado mundial de la agricultura y la alimentación. Biocombustibles: perspectivas, riesgos y oportunidades. Roma: FAO.

Herrero, Susana (2014) Análisis crítico de la seguridad alimentaria como bien público global. El caso de las poblaciones marginadas y aisladas. Madrid: UNED.

http://www.auladeeconomia.com/articulosot-14.htm accesado el 6 de enero de 2015.

Instituto Nacional de Estadística y Censos -INEC- (2014) Base de datos http:// www.ecuadorencifras.gob.ec/documentos/web-inec/Inflacion/Cambio_Anio_Base_ IPC/SIPC_20150112.pdfaccesado el 6 de enero de 2015 . 
Ministerio de Inclusión Económica y Social -MIES- (2014). Programa de Alimentación Escolar.http://educacion.gob.ec/programa-de-alimentacion-escolar/accesado el 6 de enero de 2015.

Ministerio de la Productividad -MIPRO- (2013). Política de precios para control precio alimentos. https://www.comunicacion.gob.ec/rige-en-ecuador-politica-de-preciospara-el-control-de-la-especulacion-en-46-productos-decreto/accesado el 6 de enero de 2015.

República del Ecuador (2013). Proyecto de ley orgánica de comercialización y abastecimiento alimentario. http://www.soberaniaalimentaria.gob.ec/wp-content/uploads/2013/01/ PROYECTO-DE-LEY-DE-COMERCIALIZACION-Y-ABASTECIMIENTOAGROPECUARIO-FINAL.pdf accesado el 6 de enero de 2015.

Schady, Norbert y Rosero, José (2008). Are cash transfers made to women spent like other sources of income? Economics Letters, Elsevier, vol. 101(3)

Sen, Amartya (1981). Poverty and Famines: An Essay on Entitlements and Deprivation. Oxford: Clarendon Press.

Sistema Integrado de Indicadores Sociales del Ecuador -SIISE- (2014). Base de datos. http://www.siise.gob.ec/siiseweb/siiseweb.html?sistema=1\#accesado el 6 de enero de 2015. 